\title{
Values of fractional exhaled nitric oxide for cough-variant asthma in children with chronic cough
}

\author{
Jing Zhou ${ }^{1 \#}$, Xianhong Zhao ${ }^{1 \#}$, Xu Zhang ${ }^{2,3,4 \#}$, Xingmei Yu ${ }^{1}$, Yuqing Wang ${ }^{1}$, Wujun Jiang ${ }^{1}$, Li Huang ${ }^{1}$, \\ Chuangli Hao', Luo Zhang ${ }^{2,3,4}$ \\ ${ }^{1}$ Department of Respiratory Medicine, Children's Hospital of Soochow University, Suzhou 215003, China; ${ }^{2}$ Department of Otolaryngology Head \\ and Neck Surgery, ${ }^{3}$ Department of Allergy, Beijing Tongren Hospital, Capital Medical University, Beijing 100000 , China; ${ }^{4}$ Beijing Key Laboratory of \\ Nasal Diseases, Beijing Institute of Otolaryngology, Beijing 100005, China \\ Contributions: (I) Conception and design: L Zhang, C Hao; (II) Administrative support: C Hao; (III) Provision of study materials: J Zhou, X Zhao, X \\ Zhang; (IV) Collection and assembly of data: X Yu; (V) Data analysis and interpretation: X Yu, Y Wang, W Jiang, L Huang; (VI) Manuscript writing: \\ All authors; (VII) Final approval of the manuscript: All authors. \\ \#These authors contributed equally to this work. \\ Correspondence to: Chuangli Hao. Department of Respiratory Medicine, Children's Hospital of Soochow University, Suzhou 215003, China. \\ Email: hcl_mdd@163.com; Luo Zhang. Beijing Key Laboratory of Nasal Diseases, Beijing Institute of Otolaryngology, Beijing 100005, China. \\ Email: dr.luozhang@139.com.
}

\begin{abstract}
Background: Chronic cough is a common symptom in children. We wished to explore the value of fractional exhaled nitric oxide (FeNO) for cough-variant asthma (CVA) in children with chronic cough.

Methods: This prospective cohort study was conducted in the Children's Hospital of Soochow University from January 2012 to December 2014. Children aged 6-14 years with a cough of duration $>4$ weeks were enrolled. They underwent FeNO measurement, sputum cytology and pulmonary function tests.

Results: A total of 115 patients and 25 healthy controls were evaluated. For the diagnosis of CVA, the optimal FeNO cutoff value was $25 \mathrm{ppb}$ with a sensitivity of $84.0 \%$, specificity of $97.1 \%$, positive predictive value of $97.5 \%$, and negative predictive of being $81.4 \%$. The FeNO level had a significant correlation with eosinophil count in sputum $(\mathrm{P}<0.05)$. FeNO level in CVA was decreased significantly after treatment $(\mathrm{P}=0.001)$.

Conclusions: In children, FeNO measurement might be an excellent method for diagnosing CVA with high sensitivity and specificity.
\end{abstract}

Keywords: Exhaled nitric oxide; chronic cough; cough-variant asthma (CVA); sputum eosinophils; children

Submitted Jun 05, 2018. Accepted for publication Nov 09, 2018.

doi: $10.21037 /$ jtd.2018.11.137

View this article at: http://dx.doi.org/10.21037/jtd.2018.11.137

\section{Introduction}

Cough is a common complaint in children, and can develop into chronic cough, mainly including protracted bacterial bronchitis, asthma, upper airway cough syndrome (UACS) (1). Airway inflammation is the basis for most types of chronic cough (2), and inflammatory markers in the airways are very important for the diagnosis of chronic cough. Based on this characteristic, noninvasive therapies, such as fraction of exhaled nitric oxide (FeNO) and sputum testing, have been developed to detect eosinophilic airway inflammation $(3,4)$.

Traditionally, sputum testing has been used to detect airway inflammation, but it is operator-dependent and timeconsuming. FeNO has been developed in recent years as a new technology to detect airway inflammation. Clinical evidence suggests that the FeNO level can be used to monitor airway inflammation with high specificity and sensitivity, and reflect the level of inflammatory markers in bronchoalveolar lavage fluid (BALF) (3,5). Moreover, the FeNO level is closely 
Table 1 Diagnostic criteria of chronic cough

\begin{tabular}{|c|c|}
\hline Causes & Diagnostic criteria \\
\hline Upper airway cough syndrome & (II) response to an antihistamine, nasal saline, and/or nasal steroid therapy in 2 to 4 weeks \\
\hline \multirow[t]{2}{*}{ Cough-variant asthma } & (I) An isolated chronic, nonproductive cough lasting for more than 4 weeks \\
\hline & $\begin{array}{l}\text { (II) airflow limitation demonstrated by bronchodilator responsiveness and/or response to inhaled } \\
\text { steroid (budesonide, } 400 \mu \mathrm{g} / \mathrm{d} \text { ) within } 4 \text { weeks }\end{array}$ \\
\hline \multirow{2}{*}{ Postinfectious cough } & (II) normal chest radiograph \\
\hline & (III) normal pulmonary function test results after exclusion of other causes \\
\hline \multirow[t]{3}{*}{ Eosinophilic bronchitis } & (I) Normal spirometry with normal airway responsiveness \\
\hline & (II) eosinophil count $>3 \%$ in non-squamous cell sputum \\
\hline & (III) response to glucocorticosteroids \\
\hline Tic disorders & $\begin{array}{l}\text { A neuropsychiatric disorder characterized by a waxing and waning pattern of motor and vocal tics } \\
\text { which occur several times a day, nearly every day or intermittently, over the span of more than a year }\end{array}$ \\
\hline
\end{tabular}

correlated with the results of bronchial provocation tests in patients with bronchial asthma, as well as in those infected by Lactobacillus acidophilus. Several studies have demonstrated an association between an increased FeNO level and the degree of airway inflammation. The FeNO level is increased before asthma and pulmonary dysfunction (6). One study found that the FeNO level decreased after anti-inflammatory therapy using glucocorticoids for 1 week, and is often associated with airway secretions and changes in BALF components (7). The value of using FeNO as a marker of airway inflammation in asthma has been demonstrated $(8,9)$, while its clinical importance in chronic cough has not been well studied.

In China, some studies on application of FeNO measurement in adult patients with chronic cough have been carried out (10-12). We wished to explore the potential value of FeNO measurement to diagnose cough-variant asthma (CVA) in children.

\section{Methods}

\section{Study participants and inclusion criteria}

The study protocol was approved by the Ethics Committee of Suzhou University (SZU2012-C133; Suzhou, China).
Written informed consent was obtained from all parents and children recruited into the study.

This prospective single-center study was conducted at the Children's Hospital of Soochow University (a tertiary hospital that provides services for most children in Suzhou) from January 2012 to December 2014.

Inclusion criteria were: patients aged 6-14 years with cough of duration >4 weeks; cough was the main symptom; lesions were not observed upon chest radiography; use of drugs that could affect the $\mathrm{FeNO}$ value had been stopped for $>2$ weeks.

Exclusion criteria were patients: who were reluctant to undergo FeNO measurement and pulmonary function tests; diagnosed with bronchopulmonary dysplasia, immotile cilia syndrome, tuberculosis, asthma, lung cancer, or other serious systemic diseases.

Healthy schoolchildren with normal indices of lung function and without acute respiratory infection within the previous 4 weeks were enrolled as controls.

\section{Study design}

Diagnostic criteria (Table 1) were based on clinical guidelines set by the American College of Chest Physicians for 
evaluating chronic cough in children (13). A questionnaire on drug treatment was completed. FeNO measurement was done in patients with no lesion shown on chest radiographs. Furthermore, patients underwent spirometry, sputum induction, complete blood count, differential diagnosis of common pathogens for cough. Cough score was recorded by physician. In patients with variable airflow limitation, an increase in $\mathrm{FEV}_{1}$ of $\geq 12 \%$ predicted after the administration of a bronchodilator (two puffs of albuterol administered via a mask) indicated the presence of reversible air flow limitation.

\section{Cough score}

Cough score was defined as a validated verbal category cough scale scoring daytime plus nighttime cough scores (14). This is a validated verbal category score that has been previously used in chronic cough studies in children $(14,15)$. Briefly, daytime scores were as follows: $5=$ cannot perform most usual activities due to severe coughing; 4 = frequent coughing which interferes with school or other activities; $3=$ frequent coughing but does not interfere with school or other activities; 2 = cough for more than two short periods; $1=$ cough for one or two short periods only and $0=$ no cough. Nighttime scores were as follows: $5=$ distressing cough; 4 = frequent coughs most of the night; $3=$ frequent waking due to coughing; 2 = awoken once or awoken early due to coughing; 1 = cough on waking or on going to sleep only and $0=$ no cough at night.

\section{FeNO analyses}

FeNO was measured following American Thoracic Society/ European Respiratory Society guidelines (8) using an exhaled nitric oxide analyzer $\left(\mathrm{NiOx} \mathrm{MINO}^{\circledR}\right.$; Aerocrine, Solna, Sweden). FeNO measurement was done before spirometry and sputum induction.

\section{Spirometry and bronchial provocation tests}

Vital capacity was measured using standard instrumentation (MasterScreen; Jaeger, Hoechberg, Germany) in accordance with the standards set by the European Respiratory Society (16). Forced expiratory volume in one second $\left(\mathrm{FEV}_{1}\right)$ and the $\mathrm{FEV}_{1}$ : forced vital capacity (FVC) ratio were chosen as indices for analyses. The lung function of participants with $\mathrm{FEV}_{1} \%$ predicted $>70 \%$ was assessed using a personal computer-based spirometer (microQuark; COSMED
Rome, Italy) by a quantitative jet-atomization dosing device with histamine phosphate as the excitatory drug. A bronchial provocation test was deemed "positive" if $\mathrm{FEV}_{1}$ decreased by $20 \%$ before the final step. During the test, the amount of histamine phosphate was recorded automatically. The test was defined to be "negative" if $\mathrm{FEV}_{1}$ decreased by $<15 \%$ when the maximum amount of histamine phosphate was inhaled.

\section{Sputum cytology}

When the baseline $\mathrm{FEV}_{1}$ after salbutamol inhalation was $>70 \%$ predicted, a $3 \%$ hypertonic saline solution was inhaled via an ultrasonic nebulizer for $10 \mathrm{~min}$, as described previously (17). Inhalation was discontinued if sputum was obtained or if the $\mathrm{FEV}_{1}$ decreased by $>20 \%$. Cytospin ${ }^{\mathrm{TM}}$ slides were prepared and stained with hematoxylin and eosin, and a differential cell count obtained from 400 nonsquamous cells. Only samples with cell viability $>70 \%$ and squamous-cell contamination $<20 \%$ were considered (18).

\section{Skin prick test}

Thirteen kinds (6 groups) of common aeroallergens was tested, including mites (Dermatophagoides pteronyssinus, D. farinae, and Blomia tropicalis), cockroaches (Periplaneta americana and Blattella germanica), pollens (Artemisia vulgaris, Ambrosia artemisiifolia, mixed grasses, and mixed trees), cats (Felis domesticus), dogs (Canis familiaris), and molds (mold mixes I and IV). Allergens and negative control solutions were supplied by ALK (Hørsholm, Denmark). Atopy was defined as the presence of at least one positive skin reaction to any allergen tested by skin prick test.

\section{Statistical analyses}

Statistical analyses were carried out using SPSS v19.0 (IBM, Armonk, NY, USA). Data are expressed as numbers with percentages or as the mean $\pm \mathrm{SD}$, as appropriate. The one-sample Kolmogorov-Smirnov test was used to assess the normality of data. Normally distributed continuous variables were compared using the Student's $t$-test. Analysis of variance was done to evaluate differences among groups. Based on the corresponding sensitivity and specificity at a cutoff value of $\mathrm{FeNO}$, a receiver operating curve (ROC) was constructed with 1-specificity as the abscissa and sensitivity as the ordinate. $\mathrm{P}<0.05$ was considered significant. 
Table 2 Baseline clinical characteristics of enrolled cases

\begin{tabular}{|c|c|c|c|c|c|c|}
\hline Characteristics & CVA $(n=23)$ & CVA + UACS $(n=30)$ & UACS $(n=45)$ & Other causes $(n=17)$ & Control $(n=25)$ & $P$ value \\
\hline Gender (M/F) & $12 / 11$ & $14 / 16$ & $21 / 24$ & $10 / 7$ & $14 / 11$ & 0.87 \\
\hline Body weight (kg) & $30 \pm 3$ & $27 \pm 3$ & $29 \pm 3$ & $31 \pm 3$ & $33 \pm 3$ & 0.24 \\
\hline
\end{tabular}

CVA, cough variant asthma; UACS, upper airway cough syndrome.

Table 3 Comparison of FeNO, eosinophils, pulmonary function and atopy in different diagnostic category

\begin{tabular}{|c|c|c|c|c|c|c|}
\hline Parameter & CVA $(n=23)$ & CVA + UACS $(n=30)$ & UACS $(n=45)$ & Other $(n=17)$ & Control $(n=25)$ & $P$ value \\
\hline FeNO $(p p b)^{c}$ & $38 \pm 14^{\mathrm{ab}}$ & $37 \pm 13^{\mathrm{ab}}$ & $18 \pm 7$ & $19 \pm 4$ & $13 \pm 5$ & 0.000 \\
\hline $\mathrm{FEV}_{1} \%^{\mathrm{c}}$ & $93 \pm 14$ & $92 \pm 11$ & $99 \pm 10$ & $94 \pm 10$ & $97 \pm 6$ & 0.136 \\
\hline PD20-FEV ${ }_{1}(\mathrm{mg})^{\mathrm{c}}$ & $0.5 \pm 0.2^{\mathrm{a}}$ & $0.6 \pm 0.3^{\mathrm{a}}$ & $1.5 \pm 0.7$ & $0.9 \pm 0.7$ & - & 0.001 \\
\hline Sputum eosinophils $\%^{\mathrm{c}}$ & $10.0 \pm 7.1^{\mathrm{ab}}$ & $14.7 \pm 14.5^{\mathrm{ab}}$ & $1.6 \pm 3.3$ & $0.6 \pm 0.7$ & - & 0.001 \\
\hline Atopy, n (\%) & $12(52.2)$ & $16(53.3)$ & 19 (42.2) & $6(35.3)$ & - & 0.122 \\
\hline
\end{tabular}

${ }^{a}$, compared with UACS, $\mathrm{P}<0.05 ;{ }^{\mathrm{b}}$, compared with others, $\mathrm{P}<0.05 ;{ }^{\mathrm{c}}$, data were expressed as mean $\pm \mathrm{SD}$. FeNO, fractional exhaled nitric oxide; $\mathrm{FEV}_{1}$, forced expiratory volume in one second; FVC, forced vital capacity; CVA, cough-variant asthma; UACS, upper airway cough syndrome.

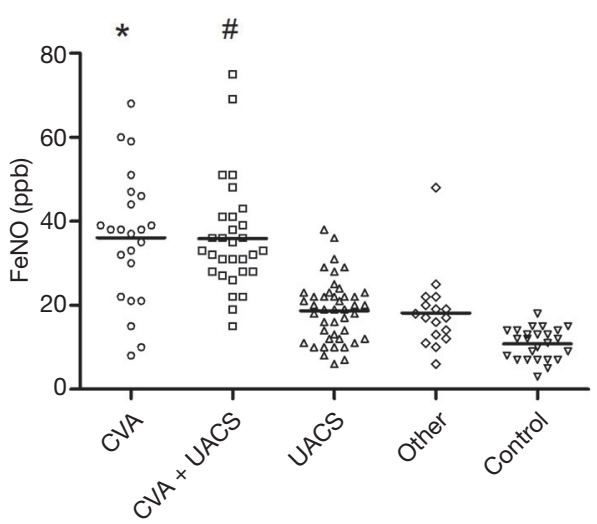

Figure 1 FeNO values by diagnostic category. FeNO values in CVA and CVA + UACS were significantly higher than those in UACS, others and the healthy control $\left({ }^{*} \mathrm{P}<0.01,{ }^{*} \mathrm{P}<0.01\right.$ respectively). FeNO, fractional exhaled nitric oxide; CVA, coughvariant asthma; UACS, upper airway cough syndrome.

\section{Results}

\section{Baseline characteristics}

A total of 127 patients met the inclusion criteria, of which 12 declined FeNO measurement. The remaining 115 cases were enrolled in our study. The 12 patients who were excluded did not differ significantly from included patients in terms of mean age at disease onset and sex.

Of these 115 patients, 23 had CVA, 30 had CVA + UACS, 45 had UACS, and 17 had other causes (PIC, GERC, and transient tic disorder). Twenty-five healthy people comprised the control group. Baseline characteristics are shown in Table 2. No significant difference between the groups in terms of age, sex, height or weight was recorded.

\section{Comparisons between FeNO levels, eosinophil count, and lung function}

FeNO levels in each group are shown in Table 3 and Figure 1. The FeNO level in the CVA group and CVA + UACS group was significantly higher than that in the UACS, other-causes, and control group $(\mathrm{P}<0.01)$. The FeNO level in the UACS group was significantly higher than that in the control group $(\mathrm{P}<0.05)$. No significant difference in the $\mathrm{FeNO}$ value was found between the CVA group and CVA + UACS group $(\mathrm{P}=0.75)$.

The eosinophil count in sputum and peripheral blood was similar among groups. The eosinophil count in the CVA group and CVA + UACS group was significantly 

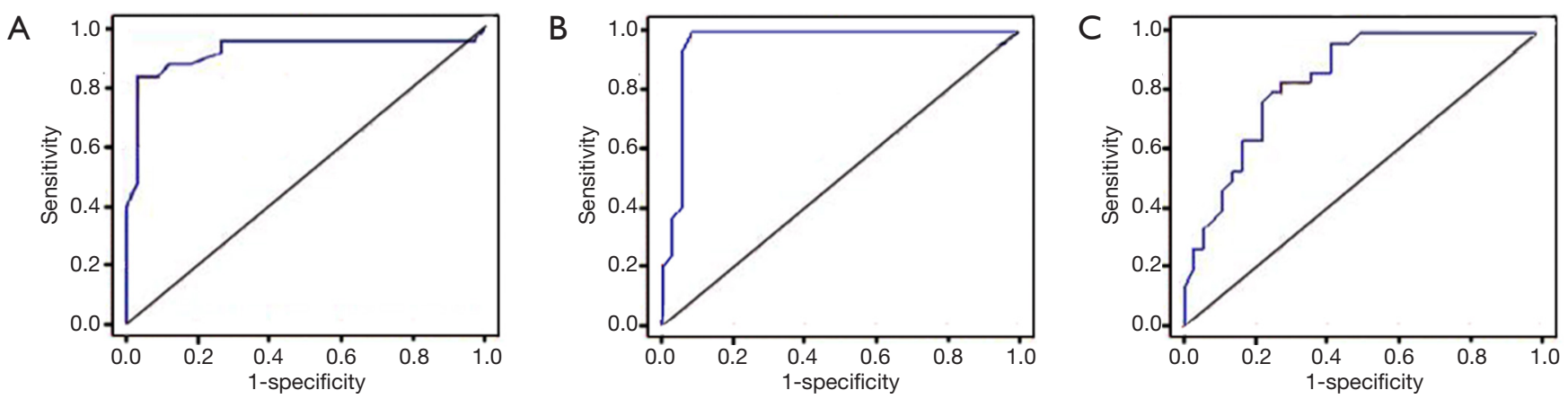

Figure 2 FeNO, sputum eosinophil and PD20 (the provocation dose required to cause a $20 \%$ reduction in FEV F $_{1}$ on CVA. (A) Diagnostic value of FeNO. The area under the ROC curve was 0.93 and the optimal cutoff level of FeNO was 25 ppb, with sensitivity of $84.0 \%$ and specificity of $97.1 \%$, for distinguishing CVA from others; (B) diagnostic value of sputum eosinophil. The area under the ROC curve was 0.96 and the optimal cutoff level of sputum eosinophil was $2 \%$, with sensitivity of $99.9 \%$ and specificity of $91.7 \%$, for distinguishing CVA from others; (C) diagnostic value of PD20. The area under the ROC curve was 0.84 and the optimal cutoff level of PD20 was 0.76 mg, with sensitivity of $83.3 \%$ and specificity of $72.2 \%$, for distinguishing CVA from others. FeNO, fractional exhaled nitric oxide; CVA, coughvariant asthma; ROC, receiver operating curve.

higher than that in the UACS group and other-causes group $(\mathrm{P}<0.05)$. No significant difference was found between the UACS group and other-causes group, or between the CVA group and CVA + UACS group, in terms of the eosinophil count (all $\mathrm{P}>0.05$ ).

The provocation dose required to cause a $20 \%$ reduction in $\mathrm{FEV}_{1}\left(\mathrm{PD} 20-\mathrm{FEV}_{1}\right)$ in the CVA group and CVA + UACS group was significantly lower than that in the UACS group $(\mathrm{P}<0.05$ for both). No significant difference was found between the CVA group and CVA + UACS group ( $\mathrm{P}=0.58)$. For $\mathrm{FEV}_{1} \%, \mathrm{FEV} 1: \mathrm{FVC}$ ratio and cough score, no significant difference was found among groups (all $\mathrm{P}>0.05$ ).

\section{CVA detection based on FeNO, eosinophil count and PD20}

As shown in Figure 2, the area under the ROC curve (AUC) was 0.93 , and the optimal sensitivity and specificity were at the cutoff value of $25 \mathrm{ppb}$. When FeNO $>25 \mathrm{ppb}$, CVA detection had a sensitivity of $84.0 \%$, specificity of $97.1 \%$, positive predictive value (PPV) of $97.5 \%$, and negative predictive value (NPV) of $81.4 \%$.

With regard to the eosinophil count in sputum, the AUC was 0.96 . An eosinophil count of $2.0 \%$ was used as the best value. When the eosinophil count was $>2.0 \%$, CVA detection had a sensitivity of $99.9 \%$, specificity of $91.7 \%$, PPV of $92.3 \%$, and NPV of $99.9 \%$.

For PD20, the AUC curve was 0.84 . PD20 of $0.76 \mathrm{mg}$ could be used as the best cutoff value. When PD20<0.76 mg, CVA detection had a sensitivity of $83.3 \%$, specificity of
$72.2 \%$, PPV of $75.0 \%$, and NPV of $81.3 \%$.

\section{Correlation between the FeNO level and PD20, eosinophil count in sputum, and lung function}

The correlation between the FeNO level and other parameters is shown in Figure 3. There was a significant positive correlation between the FeNO level and eosinophil count in sputum $(\mathrm{r}=0.362, \mathrm{P}<0.001)$, eosinophil count in peripheral blood $(\mathrm{r}=0.641, \mathrm{P}<0.641)$, but a negative correlation with the airway-reactivity parameter PD20 $(\mathrm{r}=0.411, \mathrm{P}<0.05)$. In addition, no significant correlation was found between the FeNO level and $\mathrm{FEV}_{1}(\mathrm{r}=0.124$, $\mathrm{P}>0.124)$ or $\mathrm{FEV}_{1}: \mathrm{FVC}$ ratio $(\mathrm{r}=0.027, \mathrm{P}>0.05)$.

\section{Decline of FeNO levels, eosinophils, and cough score after 4-week treatment}

FeNO level in CVA $(38 \pm 14$ versus $20 \pm 9$ ppb), CVA plus UACS $(37 \pm 13$ versus $19 \pm 6 \mathrm{ppb})$ were decreased significantly after treatment $(\mathrm{P}=0.001)$, but no significant difference was found in UACS $(18 \pm 7$ versus $17 \pm 5 \mathrm{ppb})$ and other causes group $(19 \pm 4$ versus $16 \pm 4 \mathrm{ppb})$ after the treatment, as shown in Table 4.

The ratio of sputum eosinophils in CVA group, and CVA + UACS group reduced significantly after the treatment $(\mathrm{P}=0.000)$. For the UACS group, the ratio of sputum eosinophils shows no statistically significant difference before and after the treatment. Cough score, and PD20-FEV 

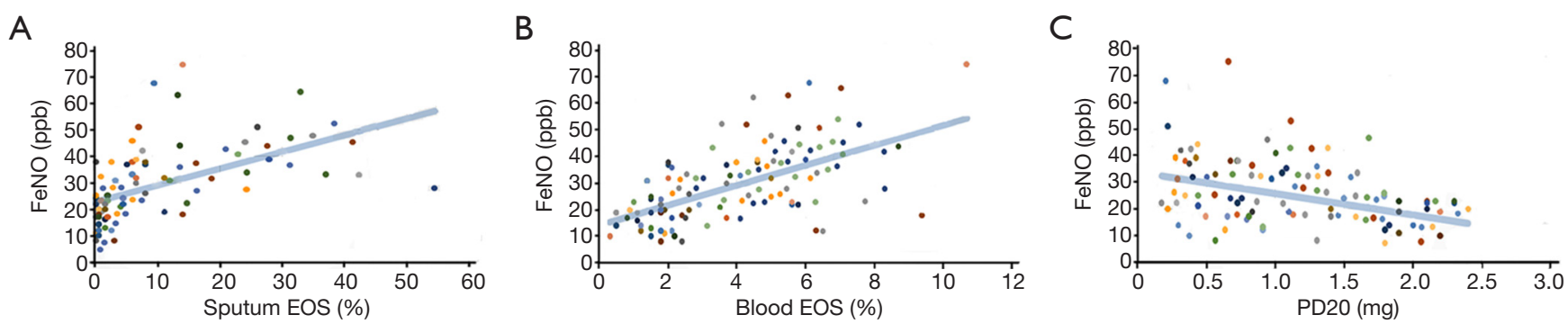

Figure 3 Correlation of FeNO values and other parameters. (A) FeNO was positively correlated with induced sputum eosinophil proportion; (B) FeNO was positively correlated with peripheral blood eosinophil proportion; (C) FeNO was negatively correlated with $\mathrm{PD} 20$ (the provocation dose required to cause a $20 \%$ reduction in $\mathrm{FEV}_{1}$ ). EOS, eosinophil; FeNO, fractional exhaled nitric oxide.

Table 4 FeNO level, sputum EOS\% and cough score comparison of before and after the 4-week treatment in children with CVA, CVA + UACS and UACS

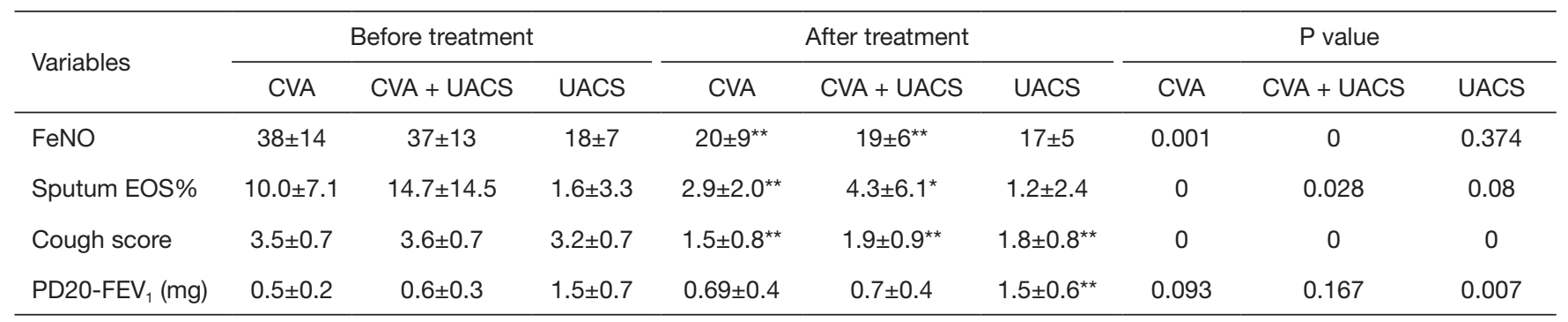

*, $\mathrm{P}<0.05$; ${ }^{* *}, \mathrm{P}<0.01$. FeNO, fractional exhaled nitric oxide; CVA, cough-variant asthma; UACS, upper airway cough syndrome; EOS, eosinophil.

level were all significantly decreased in the four groups after the treatment $(\mathrm{P}<0.01)$.

\section{Discussion}

CVA is relatively common among preschool and schoolage children $(19,20)$. Based on data from 115 children with chronic cough and 25 healthy controls, we discovered that the optimal FeNO cutoff value was $25 \mathrm{ppb}$, with a sensitivity of $84.0 \%$, specificity of $97.1 \%$, PPV of $97.5 \%$, and NPV of $81.4 \%$. Moreover, the FeNO level had a significant correlation with eosinophil count in sputum, cough symptom score, and cough score. The decrease in FeNO level was related significantly to the degree of decrease in the eosinophil count in sputum. Thus, we suggest that FeNO measurement may be useful in CVA diagnosis.

The FeNO level and eosinophil count in sputum have been recognized as important markers of inflammation $(21,22)$. We found that a diagnosis based on the FeNO level can show similar sensitivity to that of sputum testing and bronchial provocation tests. However, with respect to safety and resource consumption, FeNO measurement would be a better choice.

Several demographic and biologic factors can affect the FeNO level: smoking (23), age (24), and immunoglobulin-E level (25). In the present study, the background of enrolled cases (height, age, sex, and body mass index) was adjusted. The FeNO level of children with CVA was significantly higher than that of healthy cases and children with UACS. Similarly, Scollo and colleagues reported that the FeNO level in CVA patients was obviously higher than that of patients with cough due to other causes (26). Also, in children with CVA, the eosinophil count in sputum showed an identical trend with the FeNO level, suggesting the important role of the latter for CVA diagnosis.

Several studies have shown the high specificity and sensitivity of the FeNO level for the diagnosis of asthma. Among school-age children, the sensitivity and specificity can reach $91.4 \%$ and $92.0 \%$, respectively (27). Pérez Tarazona and co-workers demonstrated that $19 \mathrm{ppb}$ can be regarded as the threshold FeNO level for the diagnosis of 
asthma, with an AUC of 0.93 and sensitivity of $91.4 \%$ (28). Yao et al. found that $38.8 \mathrm{ppb}$ can be used as the threshold FeNO level for the diagnosis of chronic cough, with an AUC of 0.67 and sensitivity of $64.3 \%$ (29). We demonstrated that the optimal FeNO cutoff level for CVA detection can be $25 \mathrm{ppb}$, with a sensitivity of $84.0 \%$ and specificity of $97.1 \%$. These different cutoff levels might have been caused by differences in the demographic and biologic features of patients.

The present study had two main limitations. First, we included only school-age children (6-14 years) in the present study. The relationship between the FeNO level and chronic cough in younger children was not established. Second, data were obtained from a single center. Only multicenter studies can ensure precision, reduce selection bias, and increase the generalizability of the data accrued.

\section{Conclusions}

In children, FeNO measurement might be a good method for diagnosing CVA with high sensitivity and specificity.

\section{Acknowledgements}

We thank Arshad Makhdum, PhD, from LiwenBianji, Edanz Group China (www.liwenbianji.cn/ac), for editing the English text of a draft of this manuscript.

Funding: This work was supported by Social Development Projects of Jiangsu Province (to C Hao, grant number BE2016676), Key Lab of Respiratory Disease of Suzhou (to Ci Hao, grant number SZS201714), Research Project of Provincial Health and Family Planning Commission (to L Huang, grant number H201622), the Science and Technology Program of Suzhou (to W Jiang, grant number SYS201641 and L Huang, SYS201558); Beijing Natural Science Foundation (to X Zhang, grant number 7164247) and the Priming Scientific Research Foundation for the Junior Researcher in Beijing Tongren Hospital, Capital Medical University (to X Zhang, grant number 2015-YJJZZL-010).

\section{Footnote}

Conflicts of Interest: The authors have no conflicts of interest to declare.

Ethical Statement: The study protocol was approved by the Ethics Committee of Suzhou University (SZU2012-C133;
Suzhou, China). Written informed consent was obtained from all parents and children recruited into the study.

\section{References}

1. Chang AB, Robertson CF, Van PP, et al. A multicenter study on chronic cough in children: burden and etiologies based on a standardized management pathway. Chest 2012;142:943-50.

2. Chang AB, Oppenheimer JJ, Weinberger M, et al. Etiologies of Chronic Cough in Pediatric Cohorts: CHEST Guideline and Expert Panel Report. Chest 2017;152:607-17.

3. Lex C, Ferreira F, Zacharasiewicz A, et al. Airway eosinophilia in children with severe asthma: predictive values of noninvasive tests. Am J Respir Crit Care Med 2006;174:1286-91.

4. Warke TJ, Fitch PS, Brown V, et al. Exhaled nitric oxide correlates with airway eosinophils in childhood asthma. Thorax 2002;57:383-7.

5. Payne DN, Adcock IM, Wilson NM, et al. Relationship between exhaled nitric oxide and mucosal eosinophilic inflammation in children with difficult asthma, after treatment with oral prednisolone. Am J Respir Crit Care Med 2001;164:1376-81.

6. Smith AD, Cowan JO, Brassett KP, et al. Use of exhaled nitric oxide measurements to guide treatment in chronic asthma. N Engl J Med 2005;352:2163-73.

7. Deykin A, Lazarus SC, Fahy JV, et al. Sputum eosinophil counts predict asthma control after discontinuation of inhaled corticosteroids. J Allergy Clin Immunol 2005;115:720-7.

8. American Thoracic Society; European Respiratory Society. ATS/ERS recommendations for standardized procedures for the online and offline measurement of exhaled lower respiratory nitric oxide and nasal nitric oxide, 2005. Am J Respir Crit Care Med 2005;171:912-30.

9. Reddel HK, Taylor DR, Bateman ED, et al. An official American Thoracic Society/European Respiratory Society statement: asthma control and exacerbations: standardizing endpoints for clinical asthma trials and clinical practice. Am J Respir Crit Care Med 2009;180:59-99.

10. Yongming $Z$, Jiangtao $L$. The value of fractional exhaled nitric oxide in the diagnosis and treatment of chronic cough. Chin J Tuberc Respir Dis 2011;34:5.

11. Yongming $Z$, jiangtao L, Nan S, et al. Values of fractional exhaled nitric oxide in the diagnosis of chronic cough. Natl Med J China 2011;91:5. 
12. Zhu H, Yu X, Hao C, et al. The diagnostic value of the fractional exhaled nitric oxide for cough variant asthma in children. Zhonghua Jie He He $\mathrm{Hu} \mathrm{Xi} \mathrm{Za} \mathrm{Zhi}$ 2015;38:352-5.

13. Chang AB, Glomb WB. Guidelines for evaluating chronic cough in pediatrics: ACCP evidence-based clinical practice guidelines. Chest 2006;129:260S-283S.

14. Chang AB, Newman RG, Carlin JB, et al. Subjective scoring of cough in children: parent-completed vs childcompleted diary cards vs an objective method. Eur Respir J 1998;11:462-66

15. Chang AB, Robertson CF, van Asperen PP, et al. A cough algorithm for chronic cough in children: a multicenter, randomized controlled study. Pediatrics 2013;131:e1576-83.

16. Miller MR, Hankinson J, Brusasco V, et al. Standardisation of spirometry. Eur Respir J 2005;26:319-38.

17. Gibson PG, Wlodarczyk JW, Hensley MJ, et al. Epidemiological association of airway inflammation with asthma symptoms and airway hyperresponsiveness in childhood. Am J Respir Crit Care Med 1998;158:36-41.

18. Pavord ID, Pizzichini MM, Pizzichini E, et al. The use of induced sputum to investigate airway inflammation. Thorax 1997;52:498-501

19. Hannaway PJ, Hopper GD. Cough variant asthma in children. JAMA 1982;247:206-8.

20. Pender ES, Pollack CV. Cough-variant asthma in children and adults: case reports and review. J Emerg Med 1990;8:727-31.

21. Niimi A, Matsumoto H, Mishima M. Eosinophilic airway

Cite this article as: Zhou J, Zhao X, Zhang X, Yu X, Wang Y, Jiang W, Huang L, Hao C, Zhang L. Values of fractional exhaled nitric oxide for cough-variant asthma in children with chronic cough. J Thorac Dis 2018;10(12):6616-6623. doi: 10.21037/jtd.2018.11.137 disorders associated with chronic cough. Pulm Pharmacol Ther 2009;22:114-20.

22. Bateman ED, Hurd SS, Barnes PJ, et al. Global strategy for asthma management and prevention: GINA executive summary. Eur Respir J 2008;31:143-78.

23. Taylor DR, Pijnenburg MW, Smith AD, et al. Exhaled nitric oxide measurements: clinical application and interpretation. Thorax 2006;61:817-27.

24. Buchvald F, Baraldi E, Carraro S, et al. Measurements of exhaled nitric oxide in healthy subjects age 4 to 17 years. J Allergy Clin Immunol 2005;115:1130-6.

25. Cardinale F, de Benedictis FM, Muggeo V, et al. Exhaled nitric oxide, total serum $\operatorname{IgE}$ and allergic sensitization in childhood asthma and allergic rhinitis. Pediatr Allergy Immunol 2005;16:236-42.

26. Scollo M, Zanconato S, Ongaro R, et al. Exhaled nitric oxide and exercise-induced bronchoconstriction in asthmatic children. Am J Respir Crit Care Med 2000;161:1047-50.

27. Sivan Y, Gadish T, Fireman E, et al. The use of exhaled nitric oxide in the diagnosis of asthma in school children. $J$ Pediatr 2009; 155:211-6.

28. Pérez Tarazona S, Martínez Camacho RM, Alfonso Diego J, et al. Diagnostic value of exhaled nitric oxide measurement in mild asthma. An Pediatr (Barc) 2011;75:320-8.

29. Yao TC, Ou LS, Lee WI, et al. Exhaled nitric oxide discriminates children with and without allergic sensitization in a population-based study. Clin Exp Allergy 2011;41:556-64. 